\title{
On Specific ABR-markers in Psychiatry
}

\author{
Nielzen S \\ Department of Clinical Neuroscience, Lund University, Sweden
}

Corresponding author: Nielzen S, MD, PhD, Department of Clinical Neuroscience, Lund University, Sweden, E-mail:

soren.nielzen@med.lu.se

Received date: March 15, 2017; Accepted date: April 1, 2017; Published date: April 7, 2017

Copyright: @ 2017 Nielzen S. This is an open-access article distributed under the terms of the Creative Commons Attribution License, which permits unrestricted use, distribution, and reproduction in any medium, provided the original author and source are credited.

Citation: Nielzen S. On specific ABR-markers in psychiatry. Clin Psychiatry 2017, 3:1.

\section{Introduction}

In the article "Brain Stem Audiometry may supply Markers for Diagnostic and Therapeutic Control in Psychiatry" [1] our group describes a further developed ABR (Auditory Brainstem Response) method using relevant psychoacoustic complex sound stimuli, augmented dissolution of registration and enhanced techniques of analysis of the final ABR-wave [2]. The method was applied to samples of healthy subjects and different groups with psychiatric diagnoses, in this case limited to adult subjects with the diagnoses of schizophrenia and ADHD (Attentional Deficit Hyperactivity Disorder). It was shown that the three study groups could be separated with a sensitivity of 85 percent and more from each other. The results are promising for further refinement and external validation of the method [3] in order to finally constitute a means of supporting processes of diagnosing and therapy control in psychiatry. A few commentaries on the method will be presented here.

\section{Clinical Relevance}

The utility of the method in clinical contexts lies in its theoretical basis of operational definition. Operationalism defines concepts (diagnoses) by the method by which they are measured. Aberrances of the ABR-wave that can be shown to be consistent, characteristic and reproducible within a specific diagnostic group point to a common biological abnormality of the neural substrate or function of the auditory pathway and its nuclei. Revealing single differences as well as creating profiles requests a very great richness of stimuli details and a large amount of data from measuring. The analysis had been impossible, were it not for the modern computer science. Of course, it does not equate a complete psychiatric diagnosis, which is the responsibility of the clinical psychiatrist, but it may still give useful indications [4].

The schizophrenic diagnosis is often delayed due to avoidance to expose a patient to a diagnosis of a chronic illness. A support from a biological marker may then contribute to the necessity for early treatment. Effects of treatment and medication may be followed as well. It may be informative on the relative impact of multi-diagnostic influences in complicated cases. Not least is it important to asses a result typical for healthiness to avoid timeconsuming extra examinations. Certainly, a laboratory backing of a diagnostic decision may mean a corroboration of great value for the neuropsychiatric patient, his/her family, physician and related official agencies.

\section{Scientific Relevance}

For a better understanding of the neuropsychiatric disorders, the ABR-markers may be of obvious interest for researchers. The interest for mesencephalic processes and their connection with e.g. schizophrenia and autism has increased considerably in recent times. This is not surprising considering the essential function of midbrain nuclei (ambiguous, raphae nuclei, locus coeruleus etc.). The location of circuits known to be malfunctioning in schizophrenia (cf. e.g. pre-pulse inhibition response) is another reason for such interest. It is not farfetched to suspect similar structural or physiological weak spots in several psychiatric disorders. The advantage with the present operational method is that any well-defined cohort may serve as target for a research project. The limit for experimentation is not yet known, but we have e.g. seen samples of extrovert and introvert personalities to be significantly separated from each other.

\section{Advancement of Knowledge}

When interpreting the results one has to be cautious. The ABR-markers consist of anomalous areas of the curve that describe an aspect of the studied group or subject. These areas represent precise locations of the midbrain. A dysfunction at a given place may be of developmental (lesional) origin. More alternatives are possible. A lesion in the surroundings of an area may influence activity of the auditory system as well as may the massive inhibitory, facilitatory and cross-correlated activity within the brainstem. It is anticipated that the method will have a great value in future translational studies to clarify pathoanatomical and pathophysiological profiles of psychiatric disorders [5-7].

\section{References}

1. Nielzén S, Holmberg J, Sköld M, Nehlstedt S (2016) Brain Stem Audiometry may supply Markers for Diagnostic and Therapeutic Control in Psychiatry. Neuroscience Letters 632: 163-168. 
2. Tarasenko MA, Swerdlow NR, Makeig S, Braff DL, Light GA (2014) The auditory brain-stem response to complex sounds; a potential biomarker for guiding treatment of psychosis. Front Psychiatry 5: 142.

3. Claesdotter-Hybbinette, Safdarzadeh-Haghighi M, Råstam $M$, Lindvall M (2015) Abnormal brainstem auditory response in young females with ADHD. Psychiatry Res 229: 750-754.

4. Nielzén S (2017) A neurophysiological test may help diagnosing unclear cases. Acta Psychopathologica 3: 2.

5. Miron O, Ari-Even Roth D, Gabis LV, Henkin Y, Shefer S, et al. (2016) Prolonged auditory brainstem responses in infants with autism. Autism Res 9: 689-695.
6. Jafari Z, Malayeri S, Rostami R (2015) Subcortical encoding of speech cues in children with attention deficit hyperactivity disorder. Clin Neurophysiol 126: 325-332.

7. Källstrand J, Nehlstedt SF, Sköld ML, Nielzén S (2012) Lateral asymmetry and reduced forward masking effect in early brainstem auditory evoked responses in schizophrenia. Psychiatry Res 196: 188-193. 